\title{
The Social Psychology of Human-agent Interaction
}

\author{
Jonathan Gratch \\ University of Southern California, Institute for Creative Technologies \\ gratch@ict.usc.edu
}

\begin{abstract}
Designers of human-agent systems often assume that users interact with machines as if they are interacting with another person. As a consequences, fidelity to human behavior is often viewed as the gold standard for judging agent design, and theories of human social psychology are often accepted without question as a framework for informing human-agent interaction. This assumption was given strength by the pioneering work of Cliff Nass showing that many of the effects studied within social psychology seem to apply to human-machine interaction. In this talk, I will illustrate that these social effects are much weaker than widely supposed, and that the differences in how people treat machines are arguably more interesting than the similarities. These differences can lead to novel insights into human social cognition and unique technological solutions to intractable social problems. I will discuss this in the context of our research on education and mental health. Thus, rather copying human behavior, I will argue that HAI researchers should aim to transcend conventional forms of social interaction, and work towards novel theoretical frameworks that address the novel psychology of human-agent interaction.
\end{abstract}

\section{Author Keywords}

Human-centered computing, trust in autonomy, psychology of technology, affective computing

\section{BIOGRAPHY}

Jonathan Gratch is a Research Full Professor of Computer Science and Psychology at the University of Southern California's (USC) and Director for Virtual Human Research at USC's Institute for Creative Technologies. He completed his Ph.D. in Computer Science at the University of Illinois in Urban-
Champaign in 1995. Dr. Gratch's research focuses on computational models of human cognitive and social processes, especially emotion, and explores these models' role in shaping human-computer interactions in virtual environments. A recent focus of his work is on trust in autonomy and the organizational impact of algorithmic labor. $\mathrm{He}$ is the founding Editor-in-Chief of IEEE's Transactions on Affective Computing, a founding associate editor of Affective Science, and Associate Editor of Emotion Review and the Journal of Autonomous Agents and Multiagent Systems. He is an AAAI Fellow, a Cognitive Science Fellow, and ACM SIGART Autonomous Agent's Award recipient.

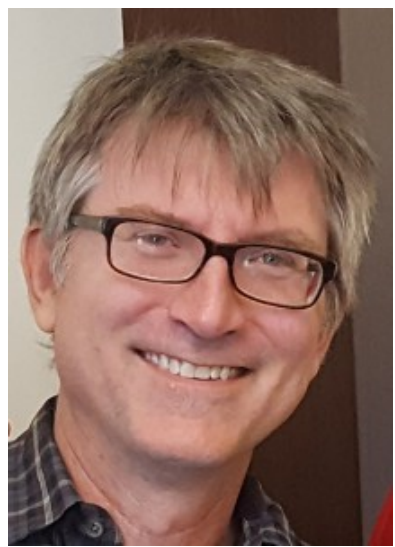

\section{ACKNOWLEDGMENTS}

This research was sponsored in part by the U.S. Army Research Laboratory under contract W911NF-14-D-0005, The Air Force Office of Scientific Research under grants FA9550-18-1-0182 and FA9550-18-0455, and the National Science Foundation under grant IIS 1822876. The views and conclusions contained in this talk are those of the author and should not be interpreted as representing the policies of any government agencies.
Permission to make digital or hard copies of part or all of this work for personal or classroom use is granted without fee provided that copies are not made or distributed for profit or commercial advantage and that copies bear this notice and the full citation on the first page. Copyrights for third-party components of this work must be honored. For all other uses, contact the Owner/Author. HAI '19, October 6-10, 2019, Kyoto, Japan.

(c) 2019 Copyright is held by the owner/author(s).

ACM ISBN 978-1-4503-6922-0/19/10.

DOI: https://doi.org/10.1145/3349537.3351909 